\title{
Overcoming Pseudomonas aeruginosa Resistance Caused by Glycocalyx with Tobracef
}

\author{
Manu Chaudhary ${ }^{1}$, Renu Bansal ${ }^{2}$ and Anurag Payasi ${ }^{1 *}$ \\ ${ }^{1}$ Department of Cell Culture and Molecular Biology, Venus Medicine Research Centre, Himachal Pradesh, India \\ ${ }^{2}$ Department of Microbiology, Faridkot Medical College, Faridkot, Punjab, India
}

\begin{abstract}
The present study was aimed to compare the binding ability of selected chemicals (adjuvants) in 23 glycocalyx positive Pseudomonas aeruginosa isolates. Fractional inhibitory concentration indexes $\left(\mathrm{FIC}_{\text {index }}\right.$ ) and drug uptake study were conducted using selected adjuvants. Whole-cell alkaline phosphatase assay was used for assessment of outer membrane permeability. FIC ${ }_{\text {index }}$ was calculated using a microdilution checkerboard method.

Among the drugs tested, Tobracef was found to be the most effective against all the selected clinical isolates with minimum inhibitory concentrations (MICs) of about 8-16 $\mu \mathrm{g} / \mathrm{ml}$. Tobracef without adjuvants showed drug uptake of approximately $85.3 \%$. When Tobracef was combined with either adjuvant $\mathrm{CH} 1$ or adjuvant $\mathrm{CH} 2$ the drug uptake was increased to $90.6 \%$ and $94.8 \%$ respectively but this increase was not statistically significant $(P>0.05)$. The drug uptake of other comparator drugs including ceftazidime, tobramycin, amikacin, gentamycin, ceftazidime plus amikacin without adjuvants varied from 14 to $34 \%$. Addition of adjuvant $\mathrm{CH} 2$ and adjuvant $\mathrm{CH} 1$ with these drugs enhanced the drug uptake by 8 to $11 \%$ and 11 to $22 \%$, respectively which was statistically significant $(P<0.01$ with adjuvant $C H 2$ and $\mathrm{P}<0.001$ with adjuvant $\mathrm{CH} 1$ ). Tobracef without adjuvants showed maximum outer membrane permeability with $8.5 \pm 0.70$ permeability index at $8 \mathrm{hrs}$. Addition of adjuvant $\mathrm{CH} 2$ or adjuvant $\mathrm{CH} 1$ into Tobracef produced $9.1 \pm 0.71$ and $9.5 \pm 0.98$ permeability index which was statistically non-significant $(P>0.05)$. Other comparator drugs exhibited a very low (only 2.4 to 3.5) permeability index and incorporation of adjuvant $\mathrm{CH} 2$ and adjuvant $\mathrm{CH} 1$ enhanced significantly the permeability index $(\mathrm{P}<0.01$ with adjuvant $\mathrm{CH} 2$ and $\mathrm{P}<0.001$ with adjuvant $\mathrm{CH} 1)$. Interestingly, all the drugs showed additive effects when tested with both the adjuvants. However the exact mechanism of high membrane permeability of Tobracef is not known which needs to be explored. Thus, Tobracef is effective in dealing resistance from $P$. aeruginosa by increasing permeability and susceptibility towards $P$. aeruginosa.
\end{abstract}

Keywords: Glycocalyx; Divalent ions; Pseudomonas aeruginosa; Clinical isolates

\section{Introduction}

Pseudomonas aeruginosa, an opportunistic gram negative pathogen, continues to be a major cause of both hospital and community acquired infections and constitutes approximately $10 \%$ of the hospital infections $[1,2]$. It is thought to be the key pathogen in patients with immune suppression, cystic fibrosis and malignancy [3]. It has been reported that more than $90 \%$ of the deaths among cystic fibrosis are caused by P. aeruginosa [4].

A characteristic feature of $P$. aeruginosa strains is production of glycocalyx, also coined as capsule when it is gelatinous in nature and slime layer when irregularly diffused, which surrounds the cells and provides a certain degree of protection for its inhabitant against environmental threats including antibiotics [5]. Glycocalyx is primarily composed of uronic acids and carbohydrates $[6,7]$ in both planktonic and biofilm cells. Glycocalyx, due to its polyanionic nature, can bind cationic antibiotics such as the aminoglycosides and thus restrict their diffusion by forming a diffusion barrier to the antimicrobial agents [8]. Amongst third generation cephalosporins, ceftazidime and amongst aminoglycoside, tobramycin are the drugs used to treat Pseudomonal infections. But in recent past, Pseudomonal resistance to these drugs has increasingly been reported $[3,9,10]$. It has been found that bacteria with glycocalyx exhibit enhanced resistant to antimicrobial agents $[11,12]$. Among the various types of mechanism of resistance, impermeability due to glycocalyx formation is the most prevalent in $P$. aeruginosa. Up to $90 \%$ of the $P$. aeruginosa isolates which are normally aminoglycoside resistant, appear to carry the impermeability type resistance [4]. It has been demonstrated that $P$. aeruginosa with glycocalyx showed a $49 \%$ decrease in permeability of tobramycin inside the bacterial membrane from $71 \%$ to $22 \%$ [13]. It has been shown that low outer membrane permeability is the root cause of all forms of resistance in $P$. aeruginosa [8]. The glycocalyx attracts divalent cations such as calcium and produce calcium complex which acts as a barrier to antimicrobial agents [14]. Thus, there is a need of an agent that breaks down the outer-membrane permeability barriers to make bacterial cells more susceptible to antibiotics [15]. It is contemplated that blocking of calcium ions may prevent the formation of glycocalyx thus use of chemicals/adjuvants with binding/blocking property together with antibiotics may be helpful in the treatment of infections caused by such type of bacteria.

Looking at the challenges posed by resistant $P$. aeruginosa to the clinicians, we aimed to compare the binding ability of selected chemicals, herein after termed as adjuvants, at different concentrations that increase bacterial cell membrane permeability which can be used together with antibiotics to fight infections caused by multidrug resistant glycocalyx positive $P$. aeruginosa isolates. Furthermore, we

*Corresponding author: Dr. Anurag Payasi, Department of Cell Culture and Molecular Biology, Venus Medicine Research Centre, Hill Top Industrial Estate, Bhatoli Kalan, Baddi, Himachal Pradesh - 173205, India, Tel: 91-1795-302068; Fax: 91-1795-302133; E-mail: ccmb@venusremedies.com

Received March 31, 2014; Accepted April 26, 2014; Published May 02, 2014

Citation: Chaudhary M, Bansal R, Payasi A (2014) Overcoming Pseudomonas aeruginosa Resistance Caused by Glycocalyx with Tobracef. J Microb Biochem Technol 6: 207-211. doi:10.4172/1948-5948.1000145

Copyright: (c) 2014 Chaudhary M, et al. This is an open-access article distributed under the terms of the Creative Commons Attribution License, which permits unrestricted use, distribution, and reproduction in any medium, provided the original author and source are credited 
also studied the drug uptake to examine the quantity of drugs inside the outer membrane of glycocalyx forming $P$. aeruginosa.

\section{Materials and Methods}

\section{Drugs}

The drugs used for the study were as follows: Tobracef (ceftazidime+tobramycin), tobramycin, amikacin, gentamycin, ceftazidime, ceftazidime along with amikacin. All these were purchased from Indian market on behalf of sponsor.

\section{Bacterial strain}

A total of 23 multidrug resistant clinical isolates of $P$. aeruginosa obtained from different parts of India were included in the study. These isolates were further re-confirmed using conventional biochemical methods [16]. P. aeruginosa ATCC 27853 was used as a control strain. Prior to use, the isolates were inoculated on Soyabean casein digest broth medium (SCDBM, Hi-Media, Mumbai, India), and incubated at $37^{\circ} \mathrm{C}$ for overnight. The overnight grown cultures were then adjusted to $10^{6} \mathrm{cfu} / \mathrm{ml}$ matching the turbidity standard of $0.5 \mathrm{Mac}-$ Farland standards with SCDBM.

\section{Glycocalyx characterization}

In order to characterize the isolates having the capability to produce glycocalyx, all the clinical isolates were subjected to qualitative test for glycocalyx production as described previously [17].

\section{Adjuvants}

All of the adjuvants, such as $\mathrm{CH} 1, \mathrm{CH} 2$, (protected as trade secret), ascorbic acid, citric acid, disodium uridine monophosphate, and boric acid were obtained from Hi-Media (Mumbai, India) and reconstituted with water for injection. Working solutions were prepared using $\mathrm{MH}$ (Mueller Hinton, Himedia, Bombay, India) broth.

\section{Minimum inhibitory concentration (MIC)}

MIC testing was performed on planktonic cultures using the two fold dilution method according to the Clinical and Laboratory Standards Institute (CLSI) guidelines [18]. The MIC value represents the lowest dilution at which bacteria fail to grow.

\section{Fractional inhibitory concentration (FIC) study}

In vitro drug interaction was determined by the checkerboard method as described elsewhere [19] and results were analyzed with the FIC $_{\text {index }}$. Growth control wells containing medium were included in each plate. Each test was performed in triplicate. The concentration of antibiotics needed to inhibit growth was recorded. The following formula was used to calculate FIC:

$$
\mathrm{FIC}=\frac{\text { MIC of drug in combination }}{\text { MIC of drug alone }}
$$

The FIC $_{\text {index }}\left(\sum\right.$ FIC) calculated as the sum of each FIC, was interpreted as follows: synergy is defined as a $\mathrm{FIC}_{\text {index }}$ of $\leq 0.5$. Antagonism is defined as a FIC $_{\text {index }}$ of $\geq 2$. An indifferent/additive effect is defined as a $\mathrm{FIC}_{\text {index }}$ of $>0.5$ to 2 or a micro dilution decrease of 1 dilution in the MIC of the one or the other drug or no change in the MIC of either of the drugs.

\section{Effect of adjuvants on binding}

Complexometric titration method was used to evaluate the chelating ability of each of adjuvant as described previously [20]. To assess the binding ability of adjuvants on $\mathrm{Ca}^{2+}$ and $\mathrm{Mg}^{2+}$ present in bacterial cells, a range of concentrations of adjuvants varying from 1 to $150 \mathrm{mM}$ was used. Briefly, to separate conical flasks, $50 \mathrm{ml}$ of bacterial culture containing various concentrations of adjuvants were added and placed all these conical flasks in incubator shaker at $37^{\circ} \mathrm{C}$ with rotating speed of $150 \mathrm{rpm}$. From each flask, an aliquot of $5 \mathrm{ml}$ was withdrawn at time intervals of 2, 4, 6, 8, 10, and $12 \mathrm{hrs}$. All aliquots were centrifuged at $7000 \mathrm{rpm}$ to pellet the cells and supernatant was used for total alginate assay. The pellets were suspended in $2 \mathrm{ml}$ of phosphate buffer saline (PBS, pH 7.2). One $\mathrm{ml}$ of each cell suspension was added to $50 \mathrm{ml}$ of conical flasks containing $6.0 \mathrm{ml}$ of $0.5 \mathrm{M}$ adjuvant $\mathrm{CH} 1,5 \mathrm{ml}$ titration buffer (combination of $7.0 \%$ ammonium chloride and $12.5 \%$ ammonia solution, $\mathrm{pH}$ 10.5) and $5 \mathrm{ml}$ of distilled water. After addition of one drop of Eriochrome black $\mathrm{T}$ indicator, the solution was titrated against $0.25 \mathrm{M} \mathrm{MgCl}_{2}$ until the appearance of pink color. A control sample was also run simultaneously to compare the binding ability of adjuvants in comparison to control. Results are presented as percentage of binding of divalent compared to total divalent ions.

\section{Total alginate analysis}

The supernatant obtained after treatment was used to analyze the released alginate comprising of uronic acid and carbohydrate by carbazole assay [21]. The optical density (OD) was read at $530 \mathrm{~nm}$. D-glucuronic acid standard of 1 to $40 \mu \mathrm{g} / \mathrm{ml}$ was prepared.

\section{Drug uptake study}

Drug uptake study was done by quantifying the amount of amines present in the sample as described earlier [22]. For experiment, $50 \mathrm{ml}$ of bacterial samples $\left(10^{6} \mathrm{cfu} / \mathrm{ml}\right)$ were treated with drugs at their half of MIC containing adjuvants and $5 \mathrm{ml}$ aliquot was withdrawn at different time intervals of 2, 4, 6, 8, 10 and 12 hrs from each treatment group. These aliquots were centrifuged at $7000 \mathrm{rpm}$ for $5 \mathrm{~min}$ and pellets were suspended in $2 \mathrm{ml}$ of $10 \mathrm{mM}$ PBS. One $\mathrm{ml}$ of each bacterial suspension was added to tubes containing $1.0 \mathrm{ml}$ of ninhydrin reagent $(1 \% \mathrm{w} / \mathrm{v})$ and $0.1 \mathrm{ml}$ of pyridine and vortexed immediately. All these tubes were then immediately placed at $70^{\circ} \mathrm{C}$ for $15 \mathrm{~min}$. After $15 \mathrm{~min}$, tubes were cooled on ice-water bath and PBS was added to bring the total volume up to $12.5 \mathrm{ml}$. Absorbance was measured at $400 \mathrm{~nm}$. Controls were also run simultaneously.

\section{Permeability assay}

Whole-cell alkaline phosphatase assay for assessment of outer membrane permeability is carried out by method detailed by Wang et al. [23]. An overnight culture of all seventeen selected $P$. aeruginosa clinical isolates grown in SCDBM were treated with antibiotics at half MIC concentration and processed as described by Wang et al. [23] An index of outer membrane permeability was calculated as A410/ $\mathrm{A} 600(\mathrm{P})$ where $\mathrm{P}$ is the concentration of para-nitrophenyl substrate.

\section{Statistical analysis}

Results are expressed as mean \pm SD values. Statistical evaluations were carried out using one way analysis of variance (ANOVA) followed by tuke'kramer multiple comparison between control and treatments groups. A value of ${ }^{\star} \mathrm{P}<0.05 ;{ }^{* *} \mathrm{P}<0.01 ;{ }^{* * *} \mathrm{P}<0.001$, compared to controls was considered significant.

\section{Results}

\section{Strain characterization and glycocalyx identification}

All the isolates were confirmed to be $P$. aeruginosa. Out of the $23 P$. 
aeruginosa isolates, 17 (74\%) were positive for glycocalyx production. These glycocalyx positive isolates were used for further study.

\section{MIC}

Among the drugs tested, Tobracef was found to be the most effective against all the selected clinical isolates with MIC values of about $16-32 \mu \mathrm{g} / \mathrm{ml}$. One fold decrease in MIC was found when Tobracef was incorporated with both adjuvant $\mathrm{CH} 2$ and adjuvant CH1. The MICs for ceftazidime and amikacin were 512-1024 and 64$128 \mu \mathrm{g} / \mathrm{ml}$, respectively. For each of tobramycin and gentamycin, MICs ranged $256-512 \mu \mathrm{g} / \mathrm{ml}$. The MIC values for ceftazidime plus amikacin were $128-256 \mu \mathrm{g} / \mathrm{ml}$. Surprisingly, when MICs of these drugs were tested with the best performing adjuvants adjuvant $\mathrm{CH} 2$ and adjuvant $\mathrm{CH} 1$, only one and two fold decrease in MIC was observed, respectively (Table 1).

\section{Effect of adjuvants on binding of divalent ions}

As expected, among the tested adjuvants adjuvant $\mathrm{CH}$ land adjuvant $\mathrm{CH} 2$ had higher binding property compared to others. Binding ability of adjuvant $\mathrm{CH} 1$ increased with increasing the concentration and reached maximum at $10 \mathrm{mM}$. Further increase in the concentration of adjuvant $\mathrm{CH} 1$ failed to yield significant binding activity. We observed that the binding ability of adjuvant $\mathrm{CH} 1$ increased with time and found to be maximum at $8 \mathrm{hrs}$ and thereafter remained almost constant up to 12 hrs (Figure 1A). Adjuvant $\mathrm{CH} 2$ also demonstrated binding activity with increasing concentration and exhibited maximum binding at $30 \mathrm{mM}$ adjuvant $\mathrm{CH} 2$ and thereafter remained constant (Figure 1B). Interestingly, adjuvant $\mathrm{CH} 2$ also showed maximum binding at $8 \mathrm{hrs}$ and then became constant. Adjuvant $\mathrm{CH} 1$ was found to be the most effective in producing highest binding ability at about three times lesser concentration of adjuvant $\mathrm{CH} 2$. The other adjuvants including ascorbic acid, citric acid, disodium uridine monophosphate, and boric acid failed to produce significant binding ability upto $150 \mathrm{mM}$ (data not shown).

\section{Effect of adjuvants on alginate reduction}

\begin{tabular}{|l|c|c|c|}
\hline \multirow{2}{*}{\multicolumn{1}{c|}{ Drugs }} & \multicolumn{3}{c|}{$\boldsymbol{P}$. aeruginosa $(\mathbf{M I C} \boldsymbol{\mu g} / \mathbf{m l})$} \\
\cline { 2 - 4 } & Without adjuvant & CH1 & CH2 \\
\hline Ceftazidime & $512-1024$ & $128-256$ & $256-512$ \\
\hline Tobramycin & $256-512$ & $64-128$ & $128-256$ \\
\hline Amikacin & $64-128$ & $16-32$ & $32-64$ \\
\hline Gentamicin & $256-512$ & $64-128$ & $128-256$ \\
\hline Ceftazidime + Amikacin & $128-256$ & $32-64$ & $64-128$ \\
\hline Tobracef & $16-32$ & 8 to16 & 8 to 16 \\
\hline
\end{tabular}

Table 1: MICs for antibacterial agents against $P$. aeruginosa clinical isolates.

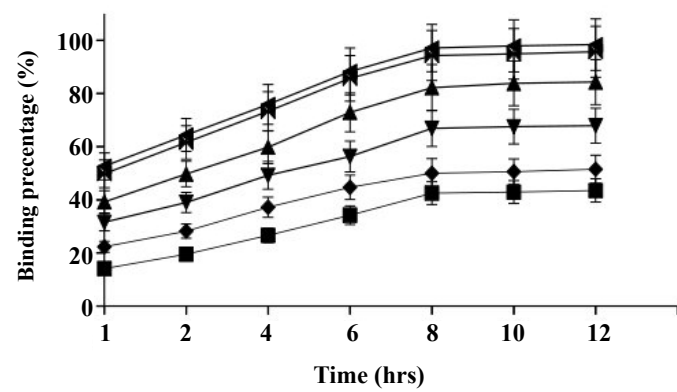

Figure 1a: Binding percentage of divalent ions by $\mathrm{CH} 1$.

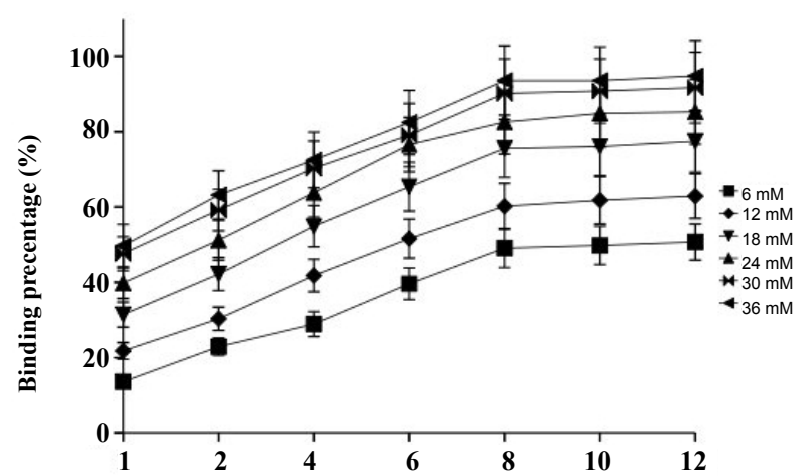

Figure 1b: Binding percentage of divalent ions by $\mathrm{CH} 2$.

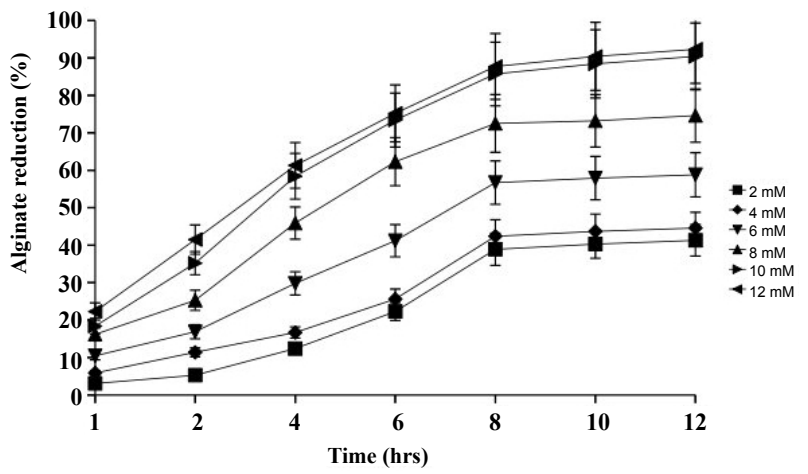

Figure 2a: Alginate reduction after $\mathrm{CH} 1$ treatment.

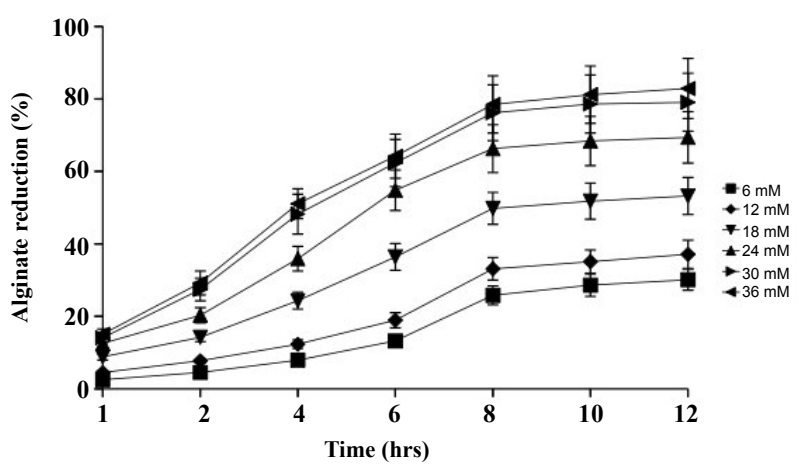

Figure 2b: Alginate reduction after $\mathrm{CH} 2$ treatment.

As shown in Figure $2 \mathrm{~A}$ and $2 \mathrm{~B}$, alginate levels reduced with increasing the concentrations of adjuvant $\mathrm{CH} 1$ and adjuvant $\mathrm{CH} 2$. Up to $85 \%$ and $76 \%$ of alginate was reduced at $8 \mathrm{hrs}$ when glycocalyx positive clinical isolates were treated with $10 \mathrm{mM}$ adjuvant $\mathrm{CH} 1$ and $30 \mathrm{mM}$ adjuvant $\mathrm{CH} 2$. Further, increasing the concentration of both of these adjuvants did not produce any significant reduction in alginate. However, the other adjuvants such as ascorbic acid, citric acid, disodium uridine monophosphate, and boric acid did not yield any pronounced reduction in alginate upto $150 \mathrm{mM}$.

\section{FIC index}

FICindex was conducted using the optimised concentrations of adjuvant $\mathrm{CH} 1(10 \mathrm{mM})$ and adjuvant $\mathrm{CH} 2(30 \mathrm{mM})$ and results are 


\begin{tabular}{|c|c|c|}
\hline \multirow[t]{2}{*}{ Drugs } & \multicolumn{2}{|c|}{ FIC index } \\
\hline & $\mathrm{CH} 2$ & $\mathrm{CH} 1$ \\
\hline Ceftazidime & $0.94 \pm 0.07$ & $0.87 \pm 0.05$ \\
\hline Tobramycin & $0.93 \pm 0.08$ & $0.88 \pm 0.06$ \\
\hline Amikacin & $0.95 \pm 0.08$ & $0.86 \pm 0.07$ \\
\hline Gentamicin & $1.43 \pm 0.09$ & $1.12 \pm 0.08$ \\
\hline Ceftazidime + Amikacin & $1.53 \pm 0.1$ & $1.13 \pm 0.07$ \\
\hline Tobracef & $1.65 \pm 0.1$ & $1.34 \pm 0.08$ \\
\hline
\end{tabular}

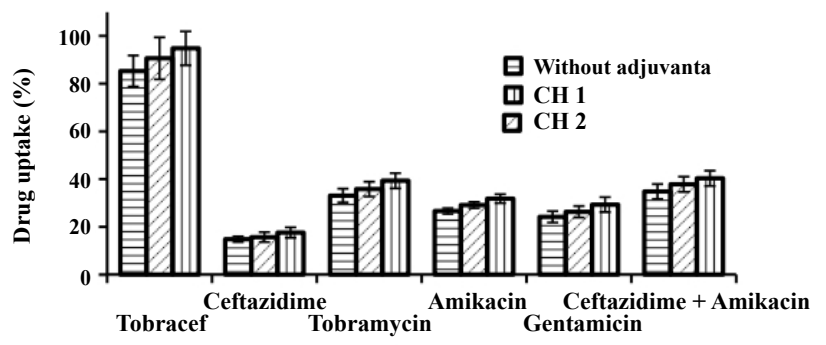

Figure 3: Comparison of drug uptake.

presented in Table 2. Results revealed that all the drugs with both adjuvants, adjuvant $\mathrm{CH} 1$ or adjuvant $\mathrm{CH} 2$ showed additive effect.

\section{Drug uptake study using ninhydrin}

After screening of the adjuvants and their concentrations which yielded highest binding, the optimized concentrations of adjuvant $\mathrm{CH} 1$ $(10 \mathrm{mM})$ and adjuvant $\mathrm{CH} 2(30 \mathrm{mM})$ was incorporated with different drugs for uptake study. We have studied the drug uptake at 2, 4, 6, 8, 10 and $12 \mathrm{hrs}$ time intervals and highest drug uptake was noted at $8 \mathrm{hrs}$ so here we have presented the data of only $8 \mathrm{hrs}$. Our results showed that Tobracef without adjuvants showed drug uptake of approximately 85.3\%. When Tobracef was combined with adjuvant $\mathrm{CH}$ lor adjuvant $\mathrm{CH} 2$ the drug uptake was increased to $90.6 \%$ and $94.8 \%$ respectively but this increase was not statistically significant $(P>0.05)$. The drug uptake of other comparator drugs including ceftazidime, tobramycin, amikacin, gentamycin, ceftazidime plus amikacin without adjuvants varied from 14 to $34 \%$. Addition of adjuvant $\mathrm{CH} 2$ and adjuvant $\mathrm{CH} 1$ with these drugs enhanced the drug uptake by 8 to $11 \%$ and 11 to $22 \%$, respectively which was statistically significant $(\mathrm{P}<0.01$ with adjuvant $\mathrm{CH} 2$ and $\mathrm{P}<0.001$ with adjuvant $\mathrm{CH} 1$ ). These results suggest that Tobracef uptake without adjuvants was highest and significantly significant $(\mathrm{P}<0.001)$ in comparison to other drugs in both cases, with or without adjuvants (Figure 3 ).

\section{Permeability assay}

To establish correlation between drug uptake and membrane permeability a whole cell alkaline phosphatase assay was performed and results were interpreted as outer membrane permeability index. An index of outer membrane permeability was calculated to know the penetration of drugs inside the bacterial membrane. Tobracef without adjuvants showed maximum outer membrane permeability with 8.5 \pm 0.70 permeability index at 8 hours. Addition of adjuvant $\mathrm{CH} 2$ or adjuvant $\mathrm{CH} 1$ into Tobracef produced $9.1 \pm 0.71$ and $9.5 \pm 0.98$ permeability index which was statistically non-significant $(\mathrm{P}>0.05)$ suggesting incorporation of adjuvants did not further increase the permeability index. Ceftazidime without adjuvant showed the least outer membrane permeability with permeability index $1.5 \pm$ 0.11 . Addition of adjuvant $\mathrm{CH} 2$ and adjuvant $\mathrm{CH} 1$ increased the permeability index to $2.4 \pm 0.21$ and $2.6 \pm 0.18$ which was statistically significant $\mathrm{P}<0.01$ and $\mathrm{P}<0.001$, respectively. Other comparator drugs exhibited permeability index 2.4 to 3.5 and incorporation of adjuvant $\mathrm{CH} 2$ and adjuvant $\mathrm{CH} 1$ enhanced significantly the permeability index $(\mathrm{P}<0.01$ with adjuvant $\mathrm{CH} 2$ and $\mathrm{P}<0.001$ with adjuvant $\mathrm{CH} 1$ ) (Figure 3 and 4$)$.

\section{Discussion}

Now-days, $P$. aeruginosa is considered a leading cause of gram negative bacterial infections especially in immuno-suppressed patients who need prolonged hospitalization [3]. The occurrence of glycocalyx in $P$. aeruginosa is well documented $[3,24]$. Our experiment demonstrated that treatment of bacterial cells with either of adjuvants adjuvant $\mathrm{CH} 1$ or adjuvant $\mathrm{CH} 2$ resulted in significant reduction of alginate. The reduced alginate would sensitize multidrug resistant $P$. aeruginosa to the antibiotic by breaking the diffusion barrier. Earlier investigation also noted that removal of the alginate from bacterial cells changes the susceptibility of them towards drugs [25].

In addition, it has been shown that divalent metal ions such as calcium present in bacterial cell also contribute to antibiotic resistance [26-28]. Our results demonstrated that exposure of bacterial cells with either adjuvant $\mathrm{CH} 1$ or adjuvant $\mathrm{CH} 2$ yielded a significant reduction of divalent ions from bacterial cells thus helping in enhancement of susceptibility. When either of these adjuvants was incorporated with drugs one to two fold reductions in MIC was observed. In contrast to other comparator drugs, Tobracef without adjuvants showed higher sensitivity to multidrug resistant clinical isolates of $P$. aeruginos $a$ which is evident by 4 to 32 time lesser MIC in comparision to comparator drugs. These lower MICs of Tobracef is directly associated with higher permeability of drugs. Tobracef may have some kind of binding property which enhanced the uptake of this drug into bacterial cells and further addition of adjuvants with this drug failed to yield significant drug uptake.

Alkaline phosphatase assay was used to evaluate outer membrane permeability as it's a convenient tool to assess bacterial outer membrane permeability. Our data have demonstrated a positive correlation between the outer membrane permeability index and bacterial susceptibility to studied drugs in glycoclayx producing MDR $P$. aeruginosa strains. Addition of adjuvants with drugs significantly $(\mathrm{P}<0.01$ to $\mathrm{P}<0.001)$ increased permeability index because adjuvants bind divalent ions as well as remove alginate thus enhancing the permeability. However, Tobracef without adjuvants exhibit very higher

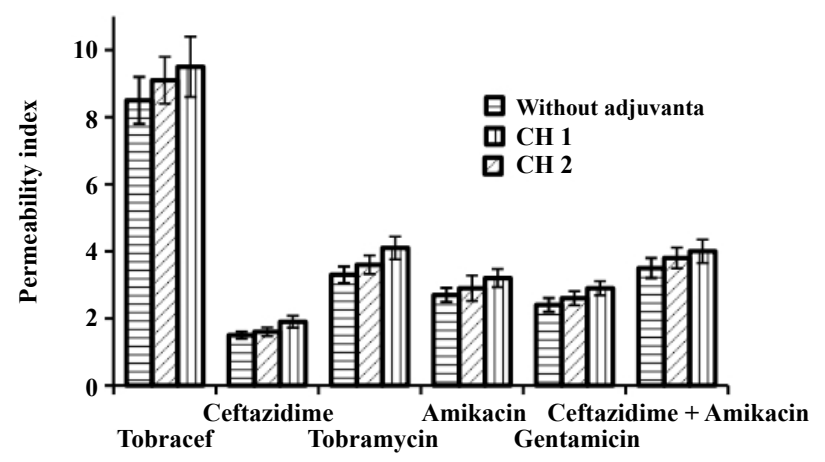

Figure 4: Comparison of bacterial outer membrane permeabilities. 
permeability index when compared with other drugs. Incorporation of adjuvant $\mathrm{CH} 2$ and adjuvant $\mathrm{CH} 1$ with Tobracef could not enhance significant permeability suggesting adjuvants failed to remove any more divalent ions or alginate from the bacterial cells and Tobracef itself is sufficient to give the activity which was several folds higher when compared with other drugs along with adjuvants because of synergistic activity.

\section{Conclusion}

Our results clearly showed that among the tested drugs Tobracef was more effective in multidrug resistant $P$. aeruginosa as evident by lesser MIC value. Also Tobracef demonstrated enhanced drug uptake and higher outer membrane permeability index compared to other drugs. However the exact mechanism of Tobracef how this causes these effects are not known which needs to be explored. Thus, Tobracef is effective in dealing resistance from $P$. aeruginosa by increasing permeability and susceptibility towards $P$. aeruginosa.

\section{Acknowledgements}

Authors are thankful to Venus Medicine Research Centre, Werne, Germany and BFMC, Faridkot, Punjab, India for providing assistance to carry out this study. Also thank to institute which provided strains.

\section{References}

1. Hancock REW, Knowles D (1998) Are we approaching the end of the antibiotic era? Editorial overview. Curr Opinion Microbiol 1: 493-494.

2. Maniatis AN, Trougakos IP, Katsanis G, Palermos J, Maniatis NA, et al. (1997) Changing patterns of bacterial nosocomial infections: a nine-year study in a general hospital. Chemotherapy 43: 69-76.

3. Arora D, Jindal N, Kumar R, Romit (2011) Emerging antibiotic resistance in Pseudomonas aeruginosa challenge. Int J Phar Pharmaceut Sci 3: 82-84.

4. Shawar RM, MacLeod DL, Garber RL, Burns JL, Stapp JR, et al. (1999) Activities of tobramycin and six other antibiotics against Pseudomonas aeruginosa isolates from patients with cystic fibrosis. Antimicrob Agents Chemother 43: 2877-2880.

5. Elder MJ, Stapleton F, Evans E, Dart JK (1995) Biofilm-related infections in ophthalmology. Eye (Lond) $9: 102-109$.

6. McAvoy MJ, Newton V, Paull A, Morgan J, Gacesa P, et al. (1989) Isolation of mucoid strains of Pseudomonas aeruginosa from non-cystic-fibrosis patients and characterization of the structure of their secreted alginate. J Med Microbio 28: 183-189.

7. Sherbrock-Cox V, Russell NJ, Gacesa P (1984) The purification and chemical characterisation of the alginate present in extracellular material produced by mucoid strains of Pseudomonas aeruginosa. Carbohydr Res 135: 147-154.

8. Nikaido $\mathrm{H}$ (1994) Prevention of drug access to bacterial targets: permeability barriers and active efflux. Science 264: 382-388.

9. Holloway WJ, Palmer D (1996) Clinical applications of a new parenteral antibiotic in the treatment of severe bacterial infections. Am J Med 100: 52S-59S.

10. Valenza G, Radike K, Schoen C, Horn S, Oesterlein A, et al. (2010) Resistance to tobramycin and colistin in isolates of Pseudomonas aeruginosa from chronically colonized patients with cystic fibrosis under antimicrobial treatment. Scand J Infect Dis 42: 885-889.

11. Anderson RL, Vess RW, Carr JH, Bond WW, Panlilio AL, et al. (1991) Investigations of intrinsic Pseudomonas cepacia contamination in commercially manufactured povidone-iodine. Infect Control Hosp Epidemiol 12: 297-302.

12. Goetz A, Muder RR (1989) Pseudomonas aeruginosa infections associated with use of povidone-iodine in patients receiving continuous ambulatory peritoneal dialysis. Infect Control Hosp Epidemiol 10: 447-450.

13. Weijmer MC, Debets-Ossenkopp YJ, Van De Vondervoort FJ, ter Wee PM (2002) Superior antimicrobial activity of trisodium citrate over heparin for catheter locking. Nephrol Dial Transplant 17: 2189-2195.

14. Jorgensen JH, Turnidge JD, Washington JA (1999) Antibacterial susceptibility tests: dilution and disk diffusion methods, p. 1526-1543, In P.R. Murray E.J. Baron, M.A. Pfaller, F.C. Tenover, and R.H. Yolken, Manual of linical microbiology, 7th ed. ASM Press, Washington, D.C.

15. Scott MG, Yan H, Hancock RE (1999) Biological properties of structurally related alpha-helical cationic antimicrobial peptides. Infect Immun 67: 20052009.

16. Kiska DL, Gilligan PH. Pseudomonas and Burkholderia. In: Murray PR Baron EJ, Pfaller MA, et al. (1995) editors. Manual of clinical microbiology. Washington, DC: American Society for Microbiology; pp. 517-25.

17. Ramsey DM, Wozniak DJ (2005) Understanding the control of Pseudomonas aeruginosa alginate synthesis and the prospects for management of chronic infections in cystic fibrosis. Mol Microbiol 56: 309-322.

18. Pereira V, Lopes C, Castro A, Silva J, Gibbs P, et al. (2009) Characterization for enterotoxin production, virulence factors, and antibiotic susceptibility of Staphylococcus aureus isolates from various foods in Portugal. Food Microbiol 26: 278-282.

19. Wijayanti MA Sholikhah EN, Hadanu $R$, Jumina J, Supargiyono $S$, et al. (2010) Additive in vitro antiplasmodial effect of N-alkyl and N-benzyl-1,10phenanthroline derivatives and cysteine protease inhibitor e64. Malar Res Treat 2010: 540786

20. Jones JD, Mcguckin WF (1964) Complexometric Titration of Calcium and Magnesium by a Semiautomated Procedure. Clin Chem 10: 767-780.

21. Dekwer D, Hempel DC (1999) Microaerophilic production of alginate by Azotobacter vinelandii. Von der Gemeinsamen Naturwissenscha ftlichen, Fakultat der Technischen UN. Carolo-Wilhelmina zu Braunschweig, Edited by Wael Sabra, aus Alexandria, Agypten 37-54

22. Thakur A, Wanchoo RK, Singh $P$ (2011) Hydrogels of Poly(acrylamide-coacrylic acid): In-vitro study on release of gentamicin sulfate. Chem Biochem Eng 25: 471-482.

23. Wang Y, Ha U, Zeng L, Jin S (2003) Regulation of membrane permeability by a two-component regulatory system in Pseudomonas aeruginosa. Antimicrob Agents Chemother 47: 95-101.

24. Dunne WM Jr (2002) Bacterial adhesion: seen any good biofilms lately? Clin Microbiol Rev 15: 155-166.

25. Diaz E, Haaf H, Lai A, and Yadana J (2011) Role of Alginate in Gentamicin Antibiotic Susceptibility during the Early Stages of Pseudomonas aeruginosa PAO1 Biofilm Establishment. J Exp Microbiol Immunol 15: 71 - 78.

26. Ramirez-Ronda CH, Holmes RK, Sanford JP (1975) Effects of divalent cations on binding of aminoglycoside antibiotics to human serum proteins and to bacteria. Antimicrob Agents Chemother 7: 239-245.

27. Campbell BD, Kadner RJ (1980) Relation of aerobiosis and ionic strength to the uptake of dihydrostreptomycin in Escherichia coli. Biochim Biophys Acta 593: 1-10.

28. Barry AL, Miller GH, Thornsberry C, Hare RS, Jones RN, et al. (1987) Influence of cation supplements on activity of netilmicin against $P$ seudomonas aeruginosa in vitro and in vivo. Antimicrob Agents Chemother 31: 1514-1518. 